\section{Standardizing distances of observations within shapes}

\section{DENNIS R. PROFFITT and RALPH G. O'BRIEN University of Virginia \\ Charlottesville, Virginia 22901}

A researcher may be interested in those responses - pointing, aiming, or eye fixation, for examplethat yield point locations within bounded shapes. $\mathrm{He}$ or she may wish to determine the relative distance of observations to some point within the shape, such as to the centroid of each shape within a set of various forms (cf. Proffitt \& Cutting, 1980; Richards \& Kaufman, 1969) or to some feature such as an eye within the contour of a face (cf. Haith, Bergman, \& Moore, 1977; Salapatek, 1975).

A problem arises because locations within any shape cannot be interpreted absolutely, but rather must be described relative to the shape in which they are located. Moreover, standardization is required to allow comparisons between observations made in different shapes. For example, consider the two shapes shown in Figure 1. Both are located on coordinate systems with centroids at the origins. Shape $A$ is an ellipse with its major axis aligned on the abscissa and its minor axis along the ordinate. Shape $B$ is a circle with a diameter equaling the length of the major axis of the ellipse. Since observations $p_{A}$ and $p_{B}$ have the same coordinate values, their absolute distances from the centroids are equivalent. However, relative to the $x$ and $y$ dimensions of the different shapes, the two observations are not equally displaced from the centroids. Observation $p_{A}$ is much closer to the boundary of the shape in the $y$ direction than in the $x$ direction, whereas $p_{B}$ is displaced from the boundary equally with respect to both coordinates. To compare observations made on the ellipse with those made on the circle, we must account for the smaller range of possible $y$ values that an observation can take within the former shape.

To achieve a standardization measure, we relate common statistical definitions to related mathematical descriptors of shapes. In particular, we relate the descriptors of a bivariate continuous density function to the moment descriptors that have previously been used in visual pattern recognition and discrimination research (cf. Alt, 1962; Hu, 1962; Zusne, 1970).

Consider a continuous bivariate distribution for

This research was supported by NIH Grant MH35530. Requests for reprints should be sent to Dennis R. Proffitt, Department of Psychology, Gilmer Hall, University of Virginia, Charlottesville, Virginia 22901. points $(\mathrm{x}, \mathrm{y})$ with density $\mathrm{f}(\mathrm{x}, \mathrm{y})$ and centroid $\left(\mu_{\mathrm{x}}, \mu_{\mathrm{y}}\right)$. The univariate density functions are

$$
g(x)=\int_{-\infty}^{+\infty} f(x, y) d y, \quad h(y)=\int_{-\infty}^{+\infty} f(x, y) d x,
$$

and the "area" (in this case, a volume) over the $(x, y)$ plane is

$$
\int_{-\infty}^{+\infty} \int_{-\infty}^{+\infty} f(x, y) d x d y=\int_{-\infty}^{+\infty} g(x) d x=\int_{-\infty}^{+\infty} h(y) d y=1 .
$$

Consider the shape depicted in Figure 2. The boundaries of the shape are equivalently defined by two pairs of continuous functions. $V_{1}(x)$ and $V_{2}(x)$ define, respectively, the upper and lower boundaries between the extreme values of $a$ and $b . W_{1}(y)$ and $W_{2}(y)$ define, respectively, the right and left boundaries between the extreme values of $c$ and $d$. For ease of presentations, only shapes definable by such boundary functions are explicitly considered here, although the principles developed for this "nice" general shape are readily adaptable to "not-so-nice" shapes as well. Typically, "not-so-nice" shapes will need to be partitioned into "nice subshapes" and solved "in pieces."

If we think of a shape as having a uniform unit density, ${ }^{1} f(x, y)=1$, then, for the general shape in Figure 2,

$$
g(x)=\int_{V_{2}(x)}^{V_{1}(x)} f(x, y) d y=V_{1}(x)-V_{2}(x)
$$

and

$$
h(y)=\int_{W_{2}(y)}^{W_{1}(y)} f(x, y) d x=W_{1}(y)-W_{2}(y) .
$$

Thus, $g(x)$ and $h(y)$ are, respectively, the height of the shape at $x$ and the width of the shape at $y$. The area of the shape is

$$
A=\int_{a}^{b} g(x) d x \equiv \int_{c}^{d} h(y) d y \equiv \int_{a}^{b} \int_{V_{2}(x)}^{V_{1}(x)} d y d x .
$$

Returning to descriptors of a statistical distribution, the variances are

$$
\sigma_{x}^{2}=\int_{-\infty}^{+\infty}\left(x-\mu_{x}\right)^{2} g(x) d x, \sigma_{y}^{2}=\int_{-\infty}^{+\infty}\left(y-\mu_{y}\right)^{2} h(y) d y
$$



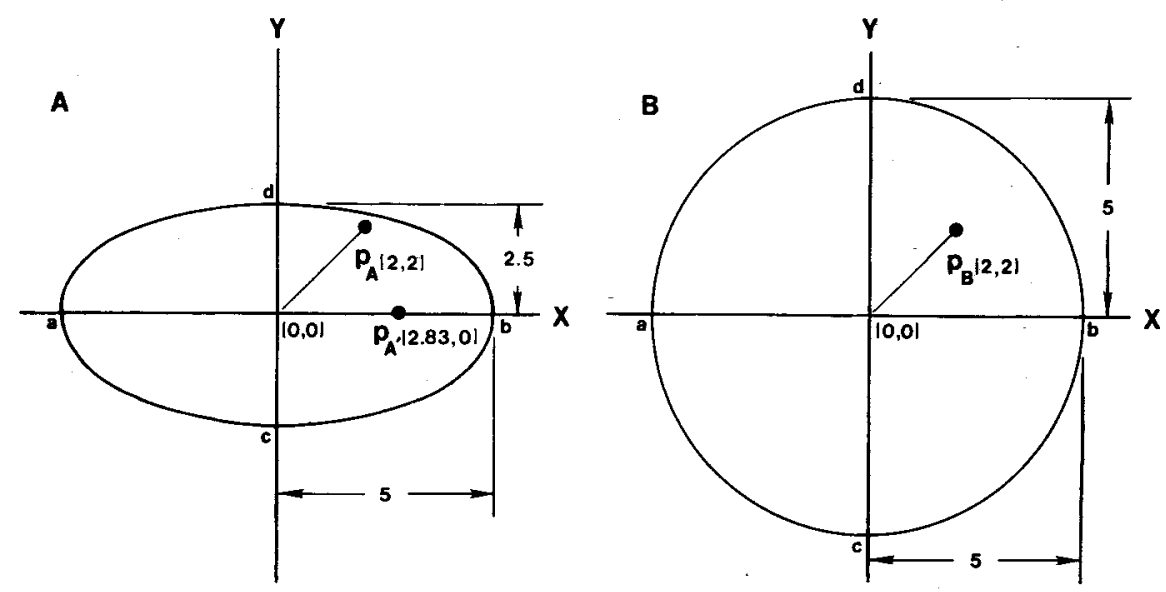

Figure 1. Two shapes are depicted with centroids located at the origins of their coordinate systems. Shape $A$ is an ellipse with its major axis aligned on the abscissa and minor axis along the ordinate. Shape $B$ is a circle of diameter equaling the length of the major axis of the ellipse. Two observations are made on shape $A, p_{A}$ and $p_{A}$, and one observation, $p_{B}$, is made on shape $B$. Although the absolute centroid relative distances of these observations are all the same, their centroid relative distances with respect to the shapes are not.

The covariance is

$$
\sigma_{x y}=\int_{-\infty}^{+\infty} \int_{-\infty}^{+\infty}\left(x-\mu_{x}\right)\left(y-\mu_{y}\right) f(x, y) d x d y
$$

which is related to the product moment correlation by

$$
\varrho_{x y}=\varrho=\sigma_{x y} / \sigma_{x} \sigma_{y} .
$$

The second moment of a shape is equivalent to the variance of a statistical distribution. Zusne (1965, 1970) has used the second moment as a description

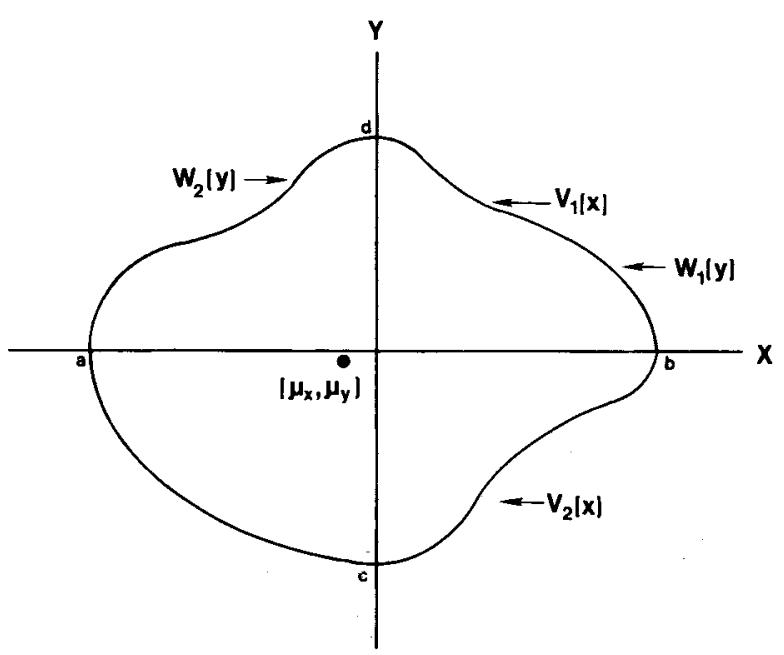

Figure 2. The shape depicted is bounded above and below by two functions of $x, V_{1}(x)$ and $V_{2}(x)$, respectively, or equivalently by two functions of $y$ bounding it to the right and left, $W_{1}(y)$ and $W_{2}(y)$, respectively. of figural "compactness," and a computer program exists (Knoll \& Stenson, 1968) for computing all area moments for shapes, including the random forms generated by the methods of Attneave and Arnoult (1956). When the boundary functions defining a shape are unknown, a procedure such as that used by Zusne (1965) can be employed. The second moments, or variances, of a shape are

$$
\begin{aligned}
& \sigma_{x}^{2}=(1 / A) \int_{a}^{b}\left(x-\mu_{x}\right)^{2} g(x) d x \\
& \sigma_{y}^{2}=(1 / A) \int_{c}^{d}\left(y-\mu_{y}\right)^{2} h(y) d y .
\end{aligned}
$$

Notice that we have normalized these variances to the area of the shape, A. This is not required in statistics, since all distributions are of unit area (or volume). Generalizing from statistics, the moment descriptor for a shape's covariance is

$$
\sigma_{x y}=(1 / A) \int_{a}^{b} \int_{V_{2}(x)}^{V_{1}(x)}\left(x-\mu_{x}\right)\left(y-\mu_{y}\right) d y d x .
$$

Having derived a set of common descriptors for both shapes and continuous bivariate statistical distributions, we can apply to the former standardization transforms developed for the latter. That is, the standardization procedures that follow apply equally to shapes and statistical distributions.

It is possible to standardize the $(x, y)$ points themselves and to standardize the distances between $(x, y)$ points. Standardizing the points uses the Z-score transform

$$
\mathrm{Z}_{\mathrm{x}}=\left(\mathrm{x}-\mu_{\mathrm{x}}\right) / o_{\mathrm{x}}, \quad \mathrm{Z}_{\mathrm{y}}=\left(\mathrm{y}-\mu_{\mathrm{y}}\right) / \sigma_{\mathrm{y}} .
$$


The absolute distance between two points, $\mathrm{p}_{1}=\left(\mathrm{x}_{1}, \mathrm{y}_{1}\right)$ and $p_{2}=\left(x_{2}, y_{2}\right)$, is

$$
d\left(p_{1}, p_{2}\right)=\left[\left(x_{1}-x_{2}\right)^{2}+\left(y_{1}-y_{2}\right)^{2}\right]^{1 / 2} .
$$

Standardized distances are employed in the discriminant analysis and classification problem (cf. Lindeman, Merenda, \& Gold, 1980, chap. 6). The standardized distance (often called the Mahalanobis distance) between $p_{1}$ and $p_{2}$ is

$$
\begin{aligned}
d_{s}\left(p_{1}, p_{2}\right)=\left\{\left[\left(Z_{x_{1}}-Z_{x_{2}}\right)^{2}+\left(Z_{y_{1}}-Z_{y_{2}}\right)^{2}\right.\right. \\
\left.\left.-2 \varrho\left(Z_{x_{1}}-Z_{x_{2}}\right)\left(Z_{y_{1}}-Z_{y_{2}}\right)\right] /\left[1-\varrho^{2}\right]\right\}^{1 / 2} .
\end{aligned}
$$

Note that for $Q=0$,

$$
d_{s}\left(p_{1}, p_{2}\right)=\left[\left(Z_{x_{1}}-Z_{x_{2}}\right)^{2}+\left(Z_{y_{1}}-Z_{y_{2}}\right)^{2}\right]^{1 / 2} .
$$

Special cases of the above distance formulas involve finding the absolute and standardized distances from any point, $\mathrm{p}=(\mathrm{x}, \mathrm{y})$, to the centroid, $\mu=\left(\mu_{\mathrm{x}}, \mu_{\mathrm{y}}\right)$, of its distribution:

$$
\begin{gathered}
\mathrm{d}(\mathrm{p}, \mu)=\left[\left(\mathrm{x}-\mu_{\mathrm{x}}\right)^{2}+\left(\mathrm{y}-\mu_{\mathrm{y}}\right)^{2}\right]^{1 / 2} \\
\mathrm{~d}_{\mathrm{s}}(\mathrm{p}, \mu)=\left[\left(\mathrm{Z}_{\mathrm{x}}^{2}+\mathrm{Z}_{\mathrm{y}}^{2}-2 \varrho \mathrm{Z}_{\mathrm{x}} \mathrm{Z}_{\mathrm{y}}\right) /\left(1-\varrho^{2}\right)\right]^{1 / 2}
\end{gathered}
$$

It is well known (to multivariate statisticians) that, for any transformation of all points $p=(x, y)$ to $\tilde{\mathbf{p}}=(\tilde{\mathbf{x}}, \tilde{\mathbf{y}})$ using

$$
\begin{aligned}
& \tilde{x}=a_{1} x+b_{1} y+c_{1}, \\
& \tilde{y}=a_{2} x+b_{2} y+c_{2},
\end{aligned}
$$

such that the transformation is nonsingular $\left(a_{1} / a_{2}\right.$ $\left.\neq b_{1} / b_{2}\right)$, the standardized distances are invariant:

$$
d_{s}\left(p_{1}, p_{2}\right)=d_{s}\left(\tilde{p}_{1}, \tilde{p}_{2}\right),
$$

and

$$
d_{s}(p, \mu)=d_{s}(\tilde{p}, \tilde{\mu}),
$$

where

$$
\tilde{\mu}=\left(a_{1} \mu_{\mathrm{x}}+\mathrm{b}_{1} \mu_{\mathrm{y}}+\mathrm{c}_{1}, \mathrm{a}_{2} \mu_{\mathrm{x}}+\mathrm{b}_{2} \mu_{\mathrm{y}}+\mathrm{c}_{2}\right) .
$$

In other words, the standardized distance is not affected by nonsingular linear transformations, even though such a transformation may alter the centroid, the scaling of the axes, and even the rotational orientation of the axes. In fact, the axes for $\tilde{x}, \tilde{y}$ will be oblique in the original $(x, y)$ space, unless the trans- formation is orthogonal $\left(a_{1} a_{2}=b_{1} b_{2}\right)$. Thus, the standardized distance is independent of any extrinsic reference frame.

To illustrate the development above, we return to Figure 1. It can be determined by the functions defining ellipses that, for shape $\mathrm{A}$,

$$
\begin{gathered}
\sigma_{\mathrm{x}}^{2}=\left[(\overline{\mathrm{ab}} / 2)^{2} / 4\right]=\left(5^{2} / 4\right)=(2.5)^{2}, \\
\sigma_{\mathrm{y}}^{2}=\left[(\overline{\mathrm{cd}} / 2)^{2} / 4\right]=\left(2.5^{2} / 4\right)=(1.25)^{2},
\end{gathered}
$$

and

$$
\sigma_{\mathrm{xy}}=0 .
$$

In like manner, the variances can be determined for the circular shape $B$ with radius $r$ :

$$
\sigma_{\mathrm{x}}^{2}=\sigma_{\mathrm{y}}^{2}=(\mathrm{r} / 2)^{2}=(2.5)^{2},
$$

and

$$
\sigma_{\mathrm{xy}}=0 .
$$

Thus, the standardized coordinates for $\mathrm{p}_{\mathrm{A}}$ and $\mathrm{p}_{\mathrm{B}}$ are

$$
\left[Z_{x_{A}}, Z_{y_{A}}\right]=[(2-0) / 2.5,(2-0) / 1.25]=[0.8,1.6]
$$

and

$$
\left[Z_{x_{B}}, Z_{y_{B}}\right]=[(2-0) / 2.5,(2-0) / 2.5]=[0.8,0.8] .
$$

The standardized distances from $p_{A}$ and $p_{B}$ to their respective centroids are

$$
\mathrm{d}_{\mathrm{s}}\left(\mathrm{p}_{\mathrm{A}}, \mu_{\mathrm{A}}\right)=\left(\mathrm{Z}_{\mathrm{x}_{\mathrm{A}}}^{2}+\mathrm{Z}_{\mathrm{y}_{\mathrm{A}}}^{2}\right)^{1 / 2}=1.79
$$

and

$$
\mathrm{d}_{\mathrm{s}}\left(\mathrm{p}_{\mathrm{B}} \mu_{\mathrm{B}}\right)=\left(\mathrm{Z}_{\mathrm{x}_{\mathrm{B}}}^{2}+\mathrm{Z}_{\mathrm{y}_{\mathrm{B}}}^{2}\right)^{1 / 2}=1.13 .
$$

The standardized distance shows that $\mathrm{p}_{\mathrm{A}}$ is relatively farther from the centroid of shape $A$ than is $p_{B}$ from the centroid of shape $B$, even though the absolute distances are the same.

To show why standardized distances should be employed when comparing points within the same shape, consider points $\mathrm{p}_{\mathrm{A}}$ and $\mathrm{p}_{\mathrm{A}^{\prime}}$ in the ellipse. Once again, their absolute distances from the mean are the same, but

$$
\begin{aligned}
\mathrm{d}_{\mathrm{s}}\left(\mathrm{p}_{\mathrm{A}^{\prime}, \mu_{\mathrm{A}}}\right) & =\left\{[(2.83-0) /(2.5)]^{2}+0^{2}\right\}^{1 / 2} \\
& =1.13<\mathrm{d}_{\mathrm{s}}\left(\mathrm{p}_{\mathrm{A}}, \mu_{\mathrm{A}}\right)=1.79 .
\end{aligned}
$$


Thus, $p_{A^{\prime}}$ is relatively closer to $\mu_{A}$ than is $p_{A}$. Note that $\mathrm{p}_{\mathrm{A}^{\prime}}$ and $\mathrm{p}_{\mathrm{B}}$ are the same standardized distance from their respective means.

To demonstrate the invariance of standardized distances under nonsingular transformations, we will compute this value for a 45-deg counterclockwise (orthogonal) rotation of the elliptical shape A. This transformation takes the form

$$
\begin{aligned}
& \tilde{\mathrm{x}}=(\sqrt{2} / 2) \mathrm{x}-(\sqrt{2} / 2) \mathrm{y}, \\
& \tilde{\mathrm{y}}=(\sqrt{2} / 2) \mathrm{x}+(\sqrt{2} / 2) \mathrm{y}
\end{aligned}
$$

The values of observation $\mathrm{p}_{\mathrm{A}}$ become $\tilde{\mathrm{p}}_{\mathrm{A}}=(0,2.83)$, and the variances, covariance, and product moment correlation are $\tilde{\sigma}_{\mathrm{x}}^{2}=\tilde{\sigma}_{\mathrm{y}}^{2}=3.91, \tilde{\sigma}_{\mathrm{xy}}=2.34$, and $\varrho=.6$. The standardized distance remains invariant:

$$
\begin{aligned}
\mathrm{d}_{\mathrm{s}}\left(\tilde{\mathrm{p}}_{\mathrm{A}}, \tilde{\mu}_{\mathrm{A}}\right) & =\left[\left(\mathrm{Z}_{\tilde{\mathrm{x}}}^{2}+\mathrm{Z}_{\tilde{\mathrm{y}}}^{2}-2 \mathrm{Z}_{\tilde{\mathrm{x}}} \mathrm{Z}_{\tilde{\mathrm{y}}}\right) /\left(1-\varrho^{2}\right)\right]^{1 / 2} \\
& =\left\{[(2.83) /(1.98)]^{2} /\left[1-.6^{2}\right]\right\}^{1 / 2} \\
& =1.79 \\
& =\mathrm{d}_{\mathrm{s}}\left(\mathrm{p}_{\mathrm{A}}, \mu_{\mathrm{A}}\right) .
\end{aligned}
$$

We have described a method for standardizing the distances between any pair of locations within any bounded shape and across the set of all bounded shapes. Taking advantage of descriptors common to shapes and statistical distributions, the method yields standardized distances that are independent of extrinsic reference frames.

\section{REFERENCES}

ALT, F. L. Digital pattern recognition by moments. In G. L. Fischer, Jr., D. K. Pollack, B. Raddack, \& M. E. Stevens (Eds.), Optical character recognition. Washington, D.C: Spartan Books, 1962.

Atrneave, F., \& Arnoult, M. D. The quantitative study of shape and pattern recognition. Psychological Bulletin, 1956, $53,452-471$.

Haith, M. M., Bergman, T., \& Moore, M. J. Eye contact and face scanning in early infancy. Science, 1977, 198, 853-854.

Hu, M.-k. A mathematical model for visual perception. In E. E. Bernard \& M. R. Kare (Eds.), Biological prototypes and synthetic systems. New York: Plenum Press, 1962.

Knole, R. L., \& Stenson, H. H. A computer program to generate and measure random forms. Perception \& Psychophysics, $1968,3,311-316$

Lindeman, R. H., Merenda, P. E., \& Gold, R. Z. Introduction to bivariate and multivariate analysis. Glenview, Ill: Scott, Foresman, 1980.

Proffitt, D. R., \& Cutting, J. E. Perceiving the centroid of curvilinearly bounded rolling shapes. Perception \& Psychophysics, 1980, 28, 484-487.

Richards, W., \& Kaufman, L. "Center-of-gravity" tendencies for fixations and flow patterns. Perception \& Psychophysics, 1969, 5, 81-84.

SalaPateK, P. Pattern perception in early infancy. In P. Salapatek \& L. B. Cohen (Eds.), Infant perception: From sensation to cognition. New York: Academic Press, 1975.

ZuSNe, L. Moments of area and of the perimeter of visual form as predictors of discrimination performance. Journal of Experimental Psychology, 1965, 69, 213-220.

Zusne, L. Visual perception of form. New York: Academic Press, 1970.

\section{NOTE}

1. This assumption is carried throughout; however, it applies only to shapes of uniform luminance. In all other cases, different assumptions must be made in determining the values of $f(x, y)$.

(Manuscript received August 10, 1981; revision accepted for publication November 16, 1981.) 